\title{
Abdominal Mass Secondary to Human Toxocariasis
}

\author{
Javad Ghoroobi, ${ }^{1}$ Leily Mohajerzadeh, ${ }^{*}$ Maliheh Khoddami, ${ }^{2}$ Alireza Mirshemirani, ${ }^{1}$ Naser Sadeghian. ${ }^{1}$ Alireza Mahdavi. ${ }^{1}$ Sayeh Hatefi
}

1 Pediatric Surgery Research Center, Shahid Beheshti University of Medical Sciences, Tehran, Iran.

2 Pediatric Pathology Research Center, Shahid Beheshti University of Medical Sciences, Tehran, Iran

\begin{abstract}
Toxocariasis is an extensive helminthic infection that leads to visceral larva migrans in humans. A 2.5-year-old girl referred for abdominal mass. She had history of pharyngitis for two weeks. There were no other symptoms. Abdominal examination revealed an irregular solid mass in right lower quadrant (RLQ). Abdominal ultrasonography revealed an echohetrogenic large mass in RLQ, liver, and retroperitoneal area. Abdominal CT scan showed a huge mass. At laparotomy a large retroperitoneal mass that involved right liver lobe, bladder, ileocecal valve, small and large intestines was found. At histopathology diagnosis of toxocariasis was made.
\end{abstract}

Key words: Toxocariasis; Human; Abdominal mass

Correspondence*: Leily Mohajerzadeh, Pediatric Surgery Research Center, Shahid Beheshti University of Medical Sciences, Tehran, Iran.

E-mail: mohajerzadehl@yahoo.com

(C) 2017, APSP J Case Rep

Submitted: 26-08-2016

Accepted: 03-12-2016

Conflict of Interest: None

Source of Support: Nil

This is an open-access article distributed under the terms of the Creative Commons Attribution License, which permits unrestricted use, distribution, and reproduction in any medium, provided the original work is properly cited.

\section{INTRODUCTION}

Toxocariasis is a human illness resulting from the dog roundworm (Toxocaracanis), the cat roundworm (Toxocaracati) or the fox (Toxocaracanis). Toxocariasis is often recognized as visceral larva migrans (VLM).[1,2] Fever, cough, wheezing, seizures, rashes, lymphadenopathy, and visual symptoms including decreased vision or blindness are its main features.[3,4] We report a case of human toxocariasis presenting with abdominal mass which was suspected as a malignancy.

\section{CASE REPORT}

A 2.5-year old girl was admitted due to an abdominal mass. She had history of pharyngitis for the last two weeks. She had no other symptoms. At physical examination an irregular solid mass was noted in RLQ approx- imate $8 \mathrm{~cm} \times 11 \mathrm{~cm}$ in size. Laboratory tests showed hemoglobin $10.1 \mathrm{gm}$ dl, with leukocytosis and $4 \%$ eosinophil. A 24-hour urine for catecholamine level was normal. LDH was 395. Abdominal ultrasonography revealed an echo-heterogenic retroperitoneal mass. CT scan showed a huge mass with mesenteric involvement. Laparotomy showed massive retroperitoneal mass involving right lobe of the liver, bladder, ileocecal valve, small and large intestines. About $50 \mathrm{~cm}$ of small intestine, cecum, appendix, ascending colon, some mesenteric lymph nodes were removed; ileostomy and colonic mucous fistula were formed. Liver and bladder biopsies were also taken (Fig.1). On microscopy, a diffuse severe chronic inflammation with multiple granulomas, including numerous eosinophils, many lymphocytes and foreign body type giant cells surrounding degenerated worm-like structures found (Fig. 2,3). These structures were also 
present in some of the foreign body type giant cells. Appendix revealed increased lymphoid follicular hyperplasia. Small intestinal wall showed acute serositis. The resected lymph nodes showed reactive changes. No evidence of malignancy was identified. The final diagnosis was severe chronic and granulomatous inflammation with degenerated structures, suggestive of parasitic infection. Serological test was positive for toxocara IgG. The patient was managed with anti-helminthic therapy. At 2-year follow-up, she has undergone ileostomy reversal and is without any mass or features of the disease.

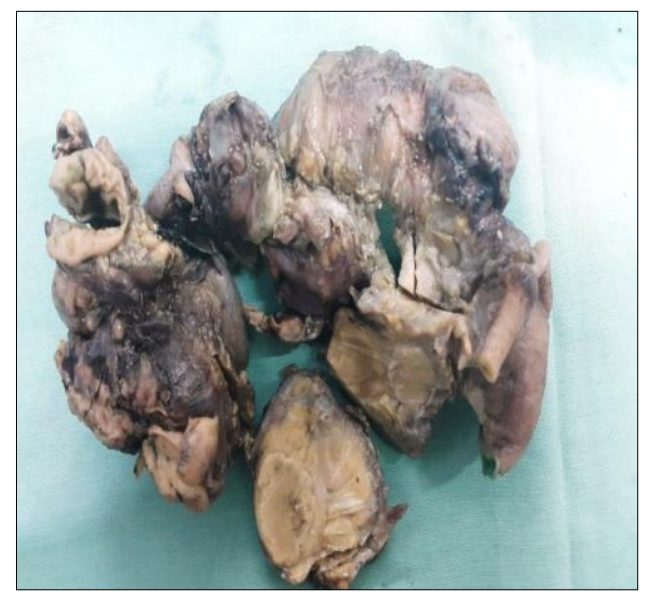

Figure 1: Three encapsulated masses with tan-yellow nodular cut surfaces in the mesocolon.

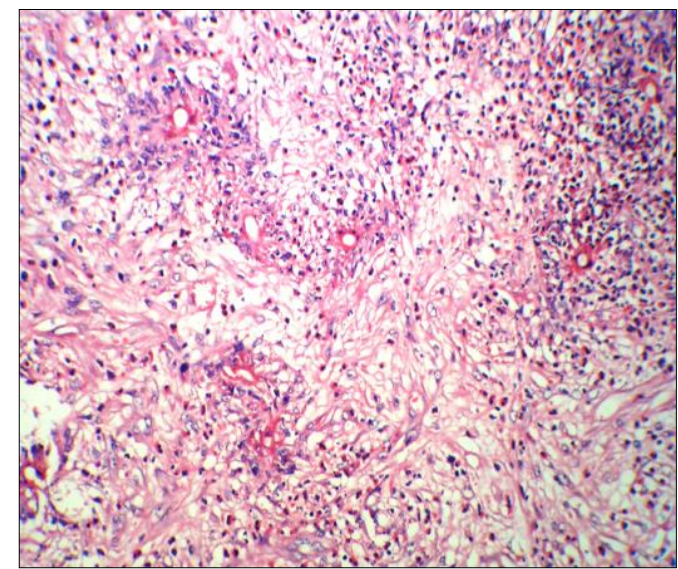

Figure 2: Microscopic examination: Severe chronic inflammation, including numerous eosinophils, many lymphocytes, and foreign body type giant cells containing degenerated worm-like structures. H\&E, X250.

\section{DISCUSSION}

Population surveys in many countries between healthy group discovered that subclinical toxocariasis is not uncommon.[2,5,6] Toxocaracanis and Toxocaracati are probably the common gastrointestinal worms (helminthes) of domestic dogs and cats and foxes. There are
3 major syndromes: visceral larva migrans (VLM), that engages major organs; covert toxocariasis, which is a milder form of VLM; and ocular larva migrans (OLM), in which eye and optic nerve are involved.[3,4] The person is infected by consuming embryonated eggs (a fully developed larva) from infected vegetables.[1] The diagnosis is usually formed after detection of Toxocara larvae.[1,2,4]. However, serological tests such as PCR, ELISA, frequently employ to identify Toxocara infection.[2,3] This worm produces granulomatous reaction in the viscera and these granulomas can be recognized by ultrasound, MRI, and CT scan as mass abdomen. Toxocariasis often heal itself, because the Toxocara larvae cannot grow-up within human hosts. Either albendazole (preferred) or mebendazole (second line therapy) may be administered.[4] Steroids can be used in case of serositis.

In our case, parasite infection, particularly VLM, had to be differentiated because visceral involvement with eosinophilia and multiple lesion on imaging were observed. We were suspecting it a neuroblastoma but exact diagnosis was guided by histopathology.

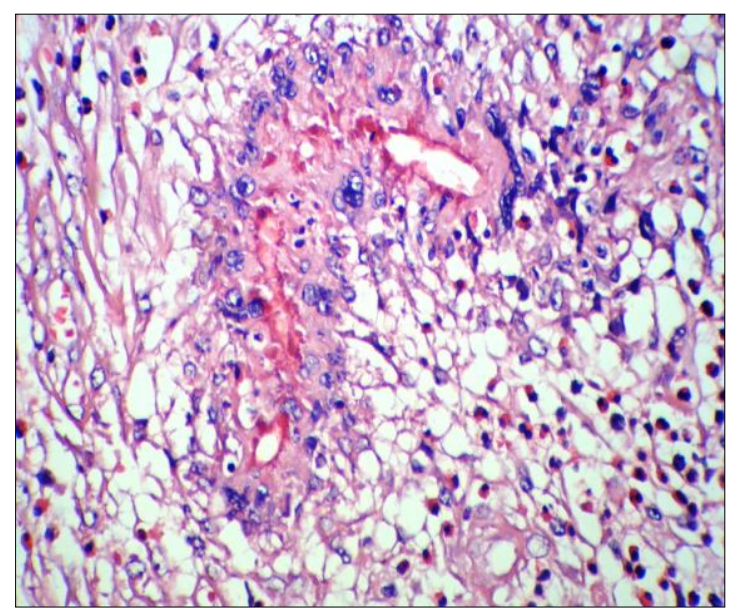

Figure 3: Microscopic examination: Degenerated worm-like structures. $\mathrm{H} \& \mathrm{E}, \mathrm{X} 400$.

\section{REFERENCES}

1. Sharghi N, Schantz PM, Hotez PJ. Toxocariasis: an occult cause of childhood asthma, seizures and neuropsychological deficit? Semin Pediatr Infect Dis. 2001; 32:E111-E6.

2. Centers for Disease Control and Prevention, Division of Parasitic diseases. "New CDC study results show Toxocara infection more common than previously thought." Available from: $\quad$ http://www.cdc.gov/ncidod/dpd/parasites/toxocara/Toxocara_announcement.pdf Accessed on. 19 Jan. 2009

3. Ming-Wei L, Rui-Qing L, Hui-Qun S, Xiang-Yun W, XingQuan Z. The complete mitochondrial genomes for three Toxocara species of human and animal health significance. BMC Genomics. 2008;9:224. 
4. Talaizadeh AH, Maraghi AS, Jelowdar A, Peyvasteh M. Human Toxocariasis: a report of 3 cases. Pak J Med Sci. 2007;23:782-4.

5. Sadjjadi SM, Khosravi M, Mehrabani D, Orya A. Seroprevalence of toxocara infection in school children in Shiraz, Southern Iran. J Trop Pediatr. 2000;46:327-30.
6. Alderete JMS, Jacob CMA, Pastorino AC, Elefant GR, Castro APM, Fomin ABF, et al. Prevalence of Toxocara Infection in Schoolchildren from the Butanta Region, Sao Paulo, Brazil. Memórias do Instituto Oswaldo Cruz. 2003;98:593-7. 